\title{
Manufacture of a Four-Sheet Complex Component from Different Titanium Alloys by Superplastic Forming
}

\author{
M. R. Allazadeh ${ }^{1, \text { a) }}$ and N. Zuelli ${ }^{1, b)}$ \\ ${ }^{1}$ Advanced Forming Research Centre (AFRC), University of Strathclyde 85 Inchinnan Drive, Inchinnan, Renfrew \\ PA4 9LJ. \\ a) Corresponding author: mohammad.allazadeh@strath.ac.uk \\ b)n.zuelli@strath.ac.uk
}

\begin{abstract}
Superplastic forming (SPF) technique was deployed to form complex component with eight-pocket features from a four-sheet sandwich panel sheetstock. Six sheetstock packs were composed of two core sheets from Ti64 or Ti54M titanium alloy and two skin sheets of Ti64 or Ti6242 titanium alloy in three different combinations. The sheets were welded by adopting two subsequent welding patterns over the core and skin sheets to satisfy the desired component's details. The applied welding methods were intermittent and continuous resistance seam welding for bonding the core sheets to each other and the skin sheets over the core panel, respectively. The final component configuration was predicted based on the dies' drawings and finite element method (FEM) simulations for the sandwich panel models. SPF system with two pipe gas feeding facilitated the SPF trials to deliver two simultaneously acting pressure-time loading curves extracted from FEM analysis for specified forming temperature and strain rate. The SPF pressure-time cycles were optimized via GOM scanning and visual observations of sections of the packs in order to assess various levels of core panel formation during the inflation process of the sheetstock to the required geometry. A GOM inspect software package compared two sets of GOM scan results, for the surface and internal features of the inflated multisheet packs, with the expected geometry of the objective component. The results suggested the capability of the demonstrated SPF method in producing complex components with novel characteristics from a flat multisheet pack out of different titanium alloys.
\end{abstract}

\section{INTRODUCTION}

Superplastic forming (SPF) is an advanced metal forming technique to expand or stretch a metal without rupture under certain forming conditions (e.g. temperature, pressure, and strain rate) well beyond its normal yielding limit by applying a differential gas pressure from at least one gas manifold. The fabrication of multisheet sandwich panel is an art within the SPF process, peculiar to certain metals, to inflate sheet metals overlaid in a sheetstock form into a complex structure with novel characteristics, including component's high-strength-to-structure-weight ratio, stiffness, corrosion resistance and usable ductility, coupled with low density and relatively low manufacture cost. Some of the applications for sandwich panel SPF technique are hollow airplane engine fan blade, wing panel, jet engine exhaust structures, access aircraft door panel, satellite fuel tank, and other honeycomb structures [1].

In the last decades, many researchers have been striving to create their own multisheets SPF technique for specific application or to obtain patent on their embodiments. Hamilton, and Ascani employed concurrent SPF and diffusion bonding (DB) techniques for the fabrication of complex variable thickness metal structures (e.g. honeycomb structures) from Ti-6A1-4V titanium alloy blank [2]. However, they utilized their manufacturing method by combining SPF/DB with the conventional manufacturing techniques to press two metal sheets as faces over a finished core panel. Froes and Eylon received patent on their embodiments for producing several titanium alloy SPF/DB components, particularly structural panels, by assembling different partially shaped parts (e.g. honeycomb, waveform, cups, patterned coatings) as core structure between two face sheets without providing the manufacturing process 
details [3]. Researchers at AFRC of the University of Strathclyde produced the fan blade using SPF/DB technique by assembling three Ti-6Al-4V sheets in which a single inner (core) was bonded to two skin sheets [4]. Kistner and Will introduced SPF with an adhesive bonding $(\mathrm{AB})$ technique to produce four sheet aluminium alloy sandwich panel structures with a pattern of internal walls parallel to one edge of the panel [5]. Velicki patented his work for fabricating a hollow core fan blade for a gas turbine engine using a four- Ti-6Al-4V sheet SPF/DB process after preparing the core structures through bending, folding, TIG welding and roll seam welding operations [6]. Hayase et. Al. invention in Mcdonnell Douglas Corporation [7] employed a biaxial intermittent or discontinuous seam weld pattern to bond four sheets of similar titanium alloy in a preselected pattern to produce the first sandwich structure with plurality of the pocket core configuration. However, they used SPF to form only the core sheets with one extension tube for the gas inlet arrangement in their embodiment, and thereof, the face sheets were placed at predefined position by the dies to obtain DB with the core sheets after they fully formed to their final shape. The main objective of aforementioned SPF works was to adapt low strain rate range but high tensile elongations deformation to minimize the necking during material flow due to inflation process. Nevertheless, many SPF multisheet technique inventors were claimed that their exemplary invention embodiments were not limited to their suggested fabricated SPF multisheet technique and cover a larger scope.

In this paper, SPF was deployed to form a complex eight-pocket sandwich panel component from four-sheet titanium alloys sheetstock. Although, the suggested sandwich panel in this paper adopted pioneering work in SPF research, it can be distinguished in this field of studies from certain manufacturing process aspects. Six flat multisheet packs were assembled from three different titanium alloys in three core-face sheets combinations to be formed under two simultaneous pressure cycle loadings. Unlike the major previous research work, the SPF process was also applied to titanium alloys other than Ti6Al4V, and multi gas pressure loading was used for forming both core and skin sheets. The SPF process was controlled by optimized pressure-time cycle loading through FEM analysis and destructive tests at $900{ }^{\circ} \mathrm{C}$.

\section{FABRICATION OF THE SHEETSTOCK}

The material chosen were Ti6Al4V, Ti6242, and Ti54M titanium alloys sheets of size $457 \mathrm{~mm}$ x $457 \mathrm{~mm}$ dimensions. The sheets were inspected visually to assure they were defect-free. The main elements in the commercial chemical composition of Ti54M are Al, V, Mo, Fe, O, for Ti6242 are Al, Zr, Mo, Sn, and for Ti64 are Al and V [8]. Thereof, the sheets have different ductility attributes which affect their manufacturing and forming processes. Each sheetstock was arranged to consist of four sheets: two core sheets from the same material, which create the inner structures of the panel; two skin sheets from the same material, which form the outer structure of the panel (Figure 1).

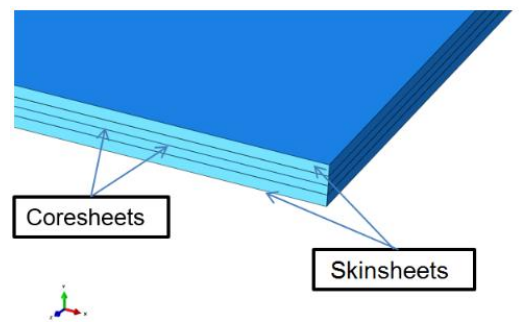

FIGURE 1. Arrangement of four sheets in the sheetstock pack.

TABLE 1. Multisheet Pack Material Configurations

\begin{tabular}{lcccc}
\hline \multirow{2}{*}{ Pack ID } & \multicolumn{2}{c}{ Core Sheets $[\mathrm{mm}]$} & \multicolumn{2}{c}{ Skin Sheets $[\mathbf{m m}]$} \\
\cline { 2 - 5 } & Material & Thickness & Material & Thickness \\
\hline P\#1, P\#2, P\#3 & Ti6Al4V & 0.8 & Ti6AL4V & 1 \\
P\#4 & Ti6Al4V & 0.8 & Ti6242 & 1 \\
P\#5, P\#6 & Ti54M & 0.625 & Ti6242 & 1 \\
\hline
\end{tabular}

Ti64 and Ti54M titanium alloys sheets were used for the core sheets, whereas Ti64 and Ti6242 titanium alloys sheets were used for the outer sheets of the multisheet packs. Each sheetstock combination was designated with a pack 
number which is shown in Table 1. After assigning the zero-point machining parameters for the machining process, all the core sheets were cut using MAXIEM 1515 water jet machine to have a V-notch at one side for internal gas inlet pipe installation. The core sheets welding pattern was designed to create eight internal separate cells between two core sheets. The inner sheets were welded together using intermittent resistance seam welding to make small gas passages between two adjacent cells. The gas passages were designed to allow the gas pressure cycle pass from the gas manifold to all cells of the core sheets during the SPF process. The outer sheets were then welded around the perimeter on either side of the already welded inner sheets except at the designated region for attaching the second gas inlet manifold to the sheetstock. Then, the second gas inlet manifold is welded to the flat packs to facilitate the second SPF pressuretime cycle loading. The length of unwelded line on the welding pattern was specified based on the hot forming die design for the inlet pipes, which was a $28 \mathrm{~mm} \times 2.5 \mathrm{~mm}$ rectangular area for both core sheets and outer sheets gas inlet pipes. Figures 2(a) and 2(b) depict the schematics of welding patterns to join the core sheets to each other and the outer sheets with the core sheets, respectively. Figure 2 also shows the location of the welding spot of the gas inlet pipes between the core sheets (Figure 2-a) and between the core panel and the external sheets in the final structure of the sheetstock (Figure 2-b). The gas pipes were welded to the sheets by TIG welding. Figure 3(a) shows an example of the welded core sheet with the inlet gas pipe. Then as it can be seen in Figure 3(b), two skin sheets are welded to the core panel according to the arrangements described in Table 1 and welding pattern of Figure 2(b) to complete the six flat multisheet packs. Argon test with very low pressure was applied to each flat pack to ensure no leakage were on the welding lines or around the inlet gas pipes.

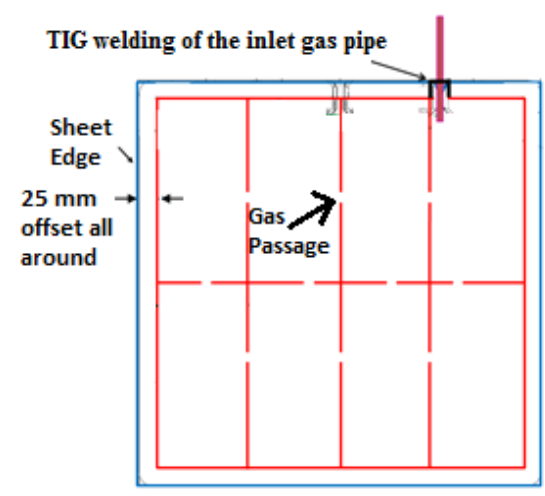

(a)

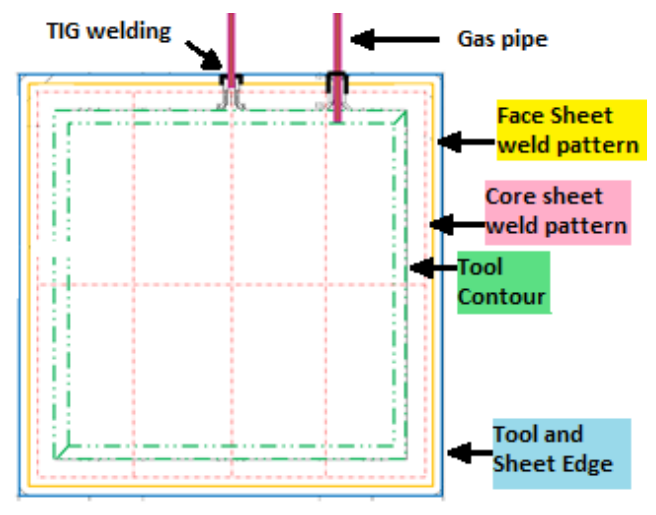

(b)

FIGURE 2. Schematic view of resistance seam welding over (a) core sheets and (b) skin sheets.

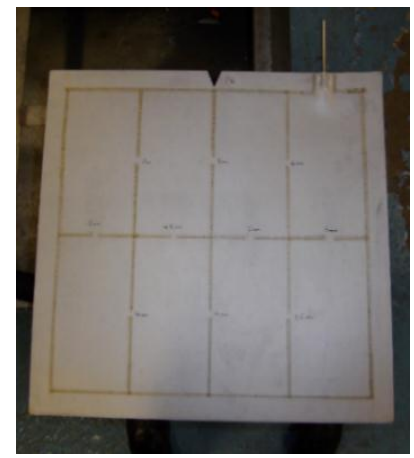

(a)

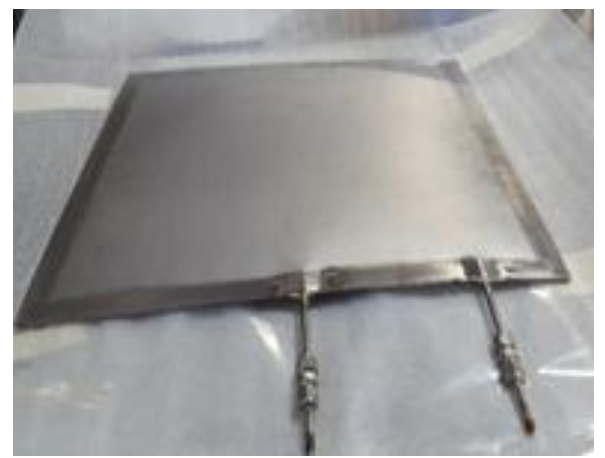

(b)

FIGURE 3. Example of the final manufactured (a) core sheet panel (b) flat multisheet pack.

\section{SPF PROCESS}

The main parameter in on SPF process is the pressure cycle defined for a desired structure which must be determined specifically for each material to be formed. The sandwich panel sheetstock and the dies were modelled in 
ABAQUS for each combination separately. Constrains of the models represent the dies surfaces, blank holders as well as welding lines over the core and skin sheets adopting the welding patterns in Figure 2. Time-hardening model in ABAQUS were used to implement the superplastic creep behaviour of materials like titanium in the simulation. Time hardening equation, also known as Bailey-Norton law, demonstrated a better curve fitting for the experimental results performed at AFRC in comparing to other built in models in ABAQUS such as the strain-hardening model and the hyperbolic sine model. The forming process involved two pressure cycles acting simultaneously through two inlet gas pipes of the flat packs to inflate the external and core sheets of the sheetstock structure, in turn. The first pressuretime cycle (Pt1) was used to blow the outer sheets into the shape of the die cavity, and the second pressure -time cycle (Pt2) was used to expand the inner sheets into the cells structure. During the forming process the pressure cycle Pt1 ramped up and once it formed the outer sheets, it decreased to zero. The pressure cycle P2 was applied until the inner sheets were in contact with the outer sheets on the die surface and the internal core structures were fully formed. Since both cycles were acting simultaneously, the pressure cycle acting on the outer sheets (Pt1) also acted against the pressure of the inner sheets. This meant that the effective inner pressure cycle is the sum of both pressure cycles. This will produce the effective inner pressure cycle to be fully formed into the inner cell structure. The pressure-time cycle for each combination of sheets listed in Table1 was initially developed using time-hardening model in ABAQUS for the target strain rate of $0.0002 \mathrm{~s}^{-1}$ at the temperature of $900{ }^{\circ} \mathrm{C}$. The SPF pressure cycles were then adjusted and optimized during the trials following GOM scanning and cutting the packs for visual inspection. To this purpose, each pack was scanned after cooling to room temperature by GOM ATOS Triple Scan III and then cut along longitudinal and transverse directions to investigate the inflation of the core sheets. Subsequently, each pressure cycle was revised based on the geometrical analysis of the packs previously formed. Therefore, six unique pressure cycles were designed for each pack regardless of their similarity in the sheets combination. Three dimensional models of the top and bottom dies designed in SolidWorks software package. A coordinate measuring machine (CMM) report was provided for each die following machining, ensuring dies symmetry and tolerances of fixing points were met. Each fixing point of the manufactured die was checked with a screw gauge following machining. Pin gauges were used to verify thermocouple port diameters of the dies. GOM scan of the dies (Figure 4) verified the manufactured die feature dimensions by comparing the dies with their design models. The colour scale in Figure 4 represents the deviation of the manufactured die from the designed model

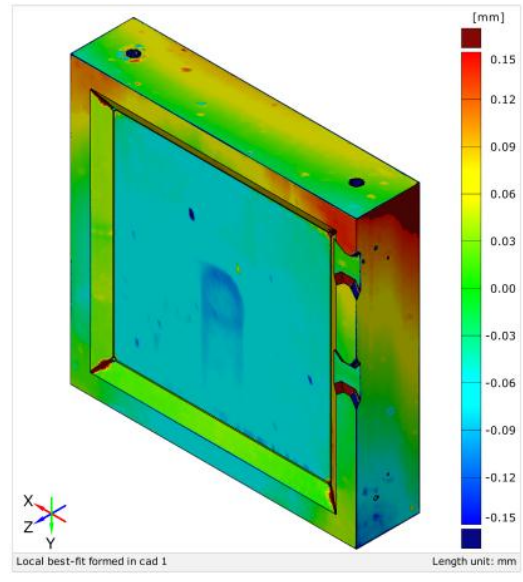

(a)

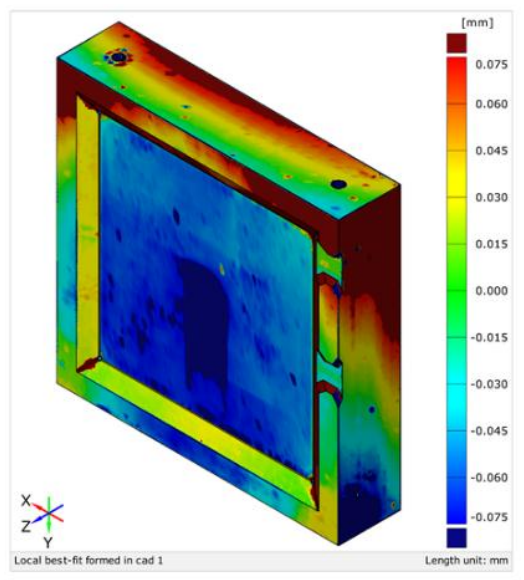

(b)

FIGURE 4 CMM measurements of (a) top and (b) bottom dies

The multisheet packs were formed in an ACB Loire 200T SPF press at the AFRC. Two pipe gas feeding SPF system facilitated the SPF trials to deliver two simultaneously acting pressure-time loading curves extracted from FEM analysis for specified forming temperature and strain rate. All surfaces of the flat packs were coated with boron nitride to prevent the sheets from sticking to the die surfaces due to compression forces at elevated temperature. All the flat packs were brought to vacuum and then pressurized to 1 bar using argon gas prior to applying the SPF pressure cycles. The ram force was set to be 30 ton based on previous SPF experiments at AFRC and to enhance the results of the diffusion bonding mechanism. A number of other SPF press parameters were necessary to be set precisely for a successful inflation, e.g. ram speed and ram position. Six thermocouples were used to control the homogeneity of the temperature inside the die cavity during the SPF process. 


\section{RESULTS AND DISCUSSIONS}

The 200T SPF press software controlled the termination parameters of the packs inflation process based on the built-in and operator's program pressure set points. The accuracy of the inflation method was examined using the GOM inspect V8 software package based on a parametric concept. The GOM inspect software generated topographic images by comparing the formed pack with the expected surface and internal features of the inflated multisheet packs, derived from design drawings and numerical simulation analysis.

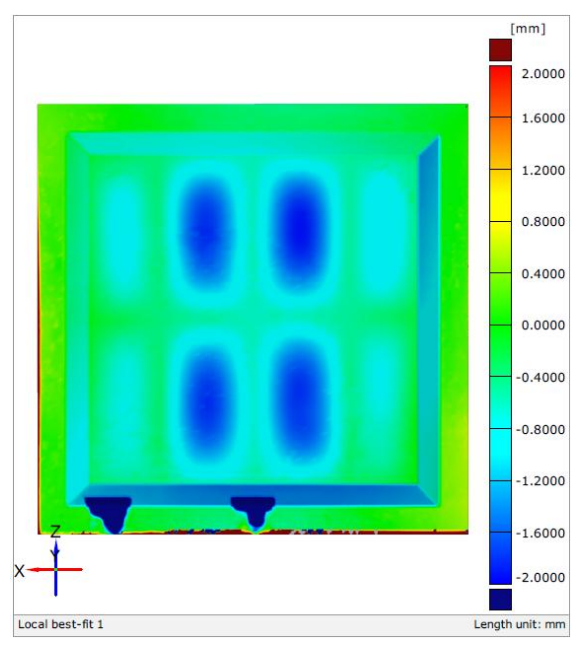

(a)

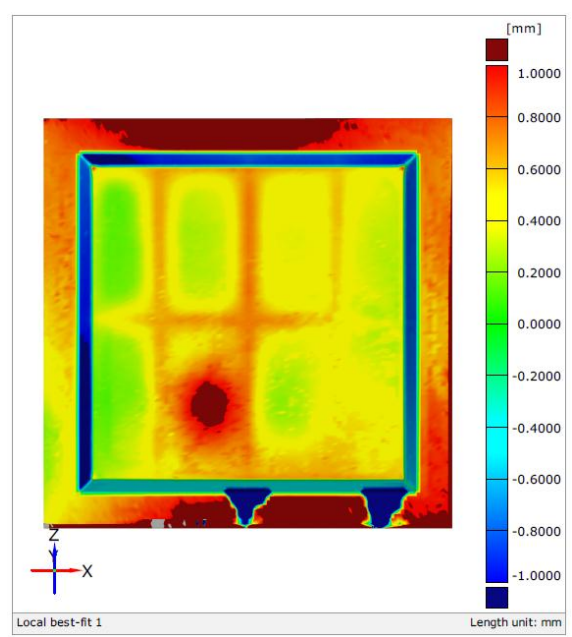

(b)

FIGURE 5. Deviation between GOM scans and FE results of the inflated multisheets packs for (a) top side of the formed pack, and (b) bottom side of the formed pack.

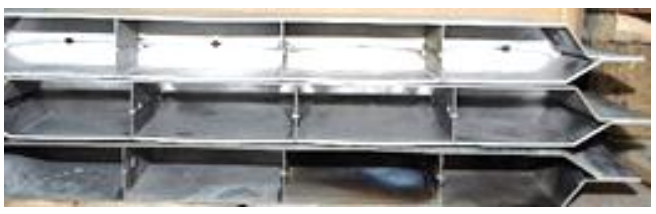

(a)

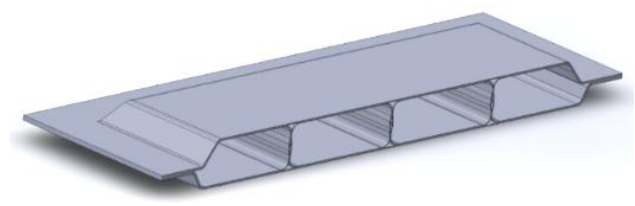

(b)

FIGURE 6. The cross-section view of the formed pack (a) after cutting and (b) from GOM results.

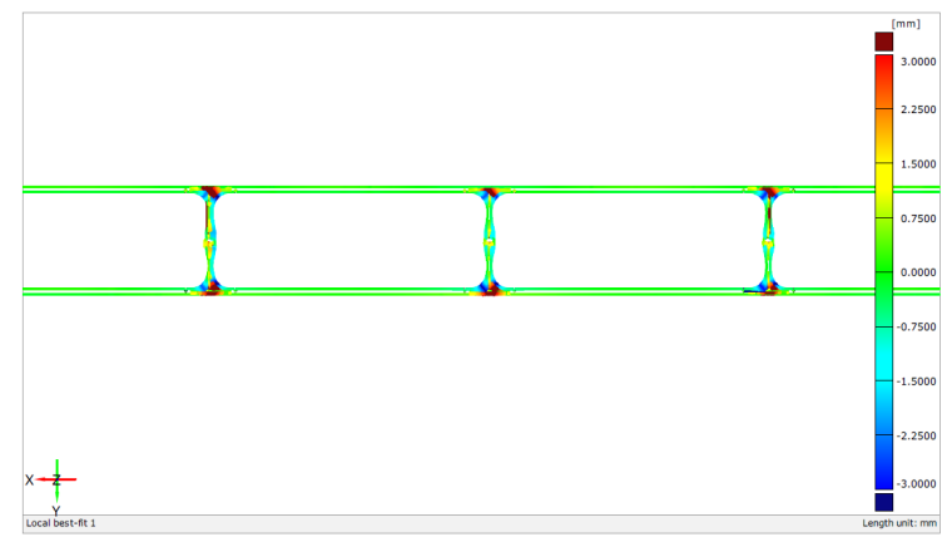

FIGURE 7. An example of GOM scan results for comparison between FE simulations results and formed packs.

Figures 5(a) and 5(b) show the deviation of the GOM scan results from the expected shape derived from FE results for the fully inflated pack of top and bottom dies, respectively. The traces of all eight internal cells in Figure 5 indicate 
possible well-formed internal cores without looking through their cross sections. The same analysis was performed for all six inflated multisheet packs. These analyses show almost the same discrepancy range between the FE and GOM scanning results. This confirmed the external sheets were formed fully according to the FE results. It was realised after cutting the packs that not all core panels fully formed. However, their shapes were very similar to the FEM simulation results for the core panel at different time steps of the simulations. Figure 6 shows a cross-section of a formed pack and highlights the internal features of the manufactured component. A second set of GOM scans was carried out on all multisheet packs after cutting transversally. Initially, several CAD drawings were produced based on the FE results (an example is shown in Figure 6-b). These drawings were then used to produce SolidWorks models to be used as a reference for comparison with the GOM scan results. Figure 7 shows the GOM scan of a cross-sections of one of the formed pack in a comparison with the FE simulations results. The scale bar is the deviation of the formed walls between the pocket due to the inflation of the core sheets from the expected shapes by FEM simulations.

\section{SUMMARY}

The SPF manufacturing process was presented for forming multisheet sandwich component composed of different titanium alloy materials for skin and core panels. SPF trials was employed to inflate six flat sheet stock to a complex eight-pocket sandwich panel component inside a pair of SPF dies. The sheetstock packs were assembled in three different combinations with two similar material core sheets from of Ti64 or Ti54M titanium alloy and two similar material skin sheets from Ti64 or Ti6242 titanium alloy. The welding patterns on the flat multisheets packs were designed precisely to produce the required final structure by means of intermittent and continuous resistance seam welding manually controlled for bonding core sheets and skin sheets, respectively. An optimized SPF process with two simultaneous pressure cycle was designed to form both skin sheets and core sheets. Time hardening creep models were embedded in FEM simulations to evaluate the final component configuration based on the dies' drawings and the sandwich panel geometry. It was deduced from the comparison between the GOM scanning analyses of the inflated packs with the FE analysis results that the implemented FE analysis was able to capture the flow of material within the admissible range of errors. The same procedure could be implemented to manufacture sandwich panels with more complex core configurations from sheetstock composed of more than three sheets. The suggested SPF process is also suitable for forming complex structure from sandwich panels out of other metal alloys with high ductile tensile characteristics under proper SPF parameters. The final results are promising in order to achieve reasonable costs and to shorten the time for manufacture complex multisheet titanium alloy components with novel characteristics.

\section{ACKNOWLEDGMENTS}

This research was supported by AFRC sponsor companies: the Boeing Corporation, TIMET, Rolls-Royce, Barnes, Bifrangi and Aubert \& Duval. We thank our colleagues at AFRC especially Jamie Frame, Craig Knowles and Kadir Paslioglu who provided insight and expertise which greatly assisted the research. Also, we appreciate the support provided by MSM Aerospace Corporation engineers and technicians whose expertise and professional cooperation enabled the manufacturing of the flat packs.

\section{REFERENCES}

1. B Cantor, H. Assender, P. Grant, Aerospace Materials, Institute of Physics Publication, Taylor\& Francis Group, LLC,13:978-1-4200-3472, (2001).

2. MCharles Howard Hamilton, Jr Leonard A Ascani, Method for superplastic forming of metals with concurrent diffusion bonding, Publication patent number:US3920175 A

3. Francis H. Froes, Daniel Eylon, Method to produce superplastically formed titanium alloy components, Publication patent number US5024369 A

4. Wood P. K. C. et. al, Materials Science Forum, DOI: 10.4028/www.scientific.net/MSF.735.215, January 2013

5. Matthew G. Kistner, Jeffrey D. Will,Multisheet sandwich panel using superplastic forming and adhesive bonding, Publication patent number: US7146727 B2

6. Alexander Velicki,SPF/DB hollow core fan blade, Publication patent numberUS5240376 A

7. Masashi Hayase, Richard C. Ecklund, Robert J. Walkington, James B. Hughes, Neil R. Williams, Metallic sandwich structure and method of fabrication, Publication patent number US4217397 A.

8. Titanium alloy guide, RMI TITANIUM COMPANY booklet. 